\title{
AUTOARRIMOS: um aplicativo para dispositivos móveis para dimensionamento de muro de arrimo por gravidade
}

AUTOARRIMOS: an application for mobile devices for dimensioning the retaining wall by gravity

\author{
Allan Silva Ferreira ${ }^{\dagger}$, Gabriel de Almeida Assumpção ${ }^{\dagger}$, Moisés Moreira de Paula ${ }^{\dagger}$, Marcelle Brandão \\ de Carvalho , Carlos Vitor de Alencar Carvalho ${ }^{\S}$
}

Como citar esse artigo. Ferreira, AS; Assumpção, GA; de Paula, MM; de Carvalho, MB; Carvalho, CVA. AUTOARRIMOS: um aplicativo para dispositivos móveis para dimensionamento de muro de arrimo por gravidade Revista Teccen. 2020 Jul./Dez.; 13 (2): 18-31.

\begin{abstract}
Resumo
É muito usual a utilização de estruturas de contenção, como muros de gravidade ou flexão, para estabilização de encostas, quando não se é possível a execução de um talude que se mantenha estável por si só, devido às características do solo. Os muros de contenção são usados para conter porções de terra próximas às edificações ou abaixo delas, a fim de garantir sua segurança contra deslizamentos do terrapleno. Os muros de gravidade são os muros de contenção que se mantém estáveis devido ao peso próprio. Tais muros têm o processo de cálculo composto por alguns passos, visto que devem ser verificadas determinadas condições de segurança e fazê-lo de modo manual torna o serviço lento. O presente trabalho visa apresentar um aplicativo para verificação de estabilidade muros de gravidade, constituídos de concreto ciclópico, com o intuito de tornar o trabalho de cálculo mais ágil, que além disso possa ser usado como ferramenta educacional. A princípio foram desenvolvidos roteiros de cálculos de seis perfis de muro, no software PTC Mathcad Express Prime 6.0. O aplicativo foi desenvolvido com a ferramenta MIT App Inventor 2, que utiliza uma programação em blocos, de interface amigável e intuitiva, o que beneficiou no desenvolvimento do aplicativo. De modo a exemplificar e testar a ferramenta, foram verificados os seis perfis, além de teste realizado por estudantes e profissionais da área. Pôde-se concluir, que ter os roteiros de cálculo proveu uma ótima base para o desenvolvimento do aplicativo, além disso o aplicativo funcionou como um bom facilitador para verificar a estabilidade de muros de gravidade, pois só solicita a escolha de perfil e a inserção de dados fundamentais, reduzindo o tempo de cálculo.

Palavras-Chave: Muro de Arrimo, Estruturas, Dispositivos Móveis, Computação Aplicada.
\end{abstract}

\begin{abstract}
More and more times the gravity or flexing walls are used for containment of ground volumes, whether to maintain the stability of slopes with construct objectives, when the project of a stable slope is not possible by itself, due to the ground characteristics. The containment walls are used for contains ground portions next of constructions or below them. in order to guarantee the security against ground slides. The gravity walls are the containment walls that stays stable due the own weight. However, these walls have the calculation divided in many steps, since safety conditions must be checked, and made this sizing work manually can convert this work in a true headache. In this article will be presented an app to verify the stability of gravity walls, made of cyclopic concrete, with the goal of making the gravity wall sizing a simple work, that in addition can also used how an educational device. First, were developed a standard calculation route for six wall profiles with the software PTC Mathcad Express Prime 6.0. The app was developed using the tool MIT App Inventor 2, this program utilizes the block programming, with friendly and intuitive interface, what benefited for the app creation. In order to exemplify and test a tool, the profiles were designed in the application and students and professionals in the field have tested the application. It can be concluded, that have the calculation scripts provided the perfect base for the app development, besides that the app will operate like a huge facilitator for the sizing of gravity walls and with extremely precision, since you only need choose the layout wall between the presented layouts and after enter with the requested main data, reducing the calculation time.

Keywords: Retaining Wall, Structures, Mobile Devices, Applied Computing.
\end{abstract}

\section{Introdução}

Devido ao avanço da urbanização e a falta de planejamento urbano surgem taludes instáveis, acarretando deslizamentos e desastres; tais eventos podem ocasionar perdas materiais e no pior dos casos, mortes. Os muros de contenção, também chamados muros de arrimo, são estruturas que impedem que essas tragédias ocorram.

Conforme Silva et al (2019) os muros de arrimo têm o processo de cálculo composto por alguns passos, visto que devem ser verificadas determinadas condições

\footnotetext{
Afiliação dos autores:
}

$\uparrow$ Graduando em Engenharia Civil pela Universidade de Vassouras, Vassouras, Rio de Janeiro, Brasil.

\$ Me. em Engenharia Civil e Docente pela Universidade de Vassouras, Vassouras, Rio de Janeiro, Brasil.

$\S$ Dr. em Engenharia Civil e Docente pela Universidade de Vassouras, Vassouras, Rio de Janeiro, Brasil. 
de segurança e fazê-lo de modo manual demanda muito tempo, além disso existem poucas ferramentas para tal finalidade, o que justifica a idealização deste trabalho.

Assim, o objetivo do presente trabalho é desenvolver formas de se otimizar o processo de cálculo, partindo da hipótese de que tal processo pode ser facilitado com uso da informática. Daí surgiram os questionamentos: dimensionar quais tipos de muro? Como otimizar o processo? Quais ferramentas utilizar? Qual relevância pode ter o trabalho?

Delimitou-se o desenvolvimento do trabalho nas seguintes etapas: pesquisa bibliográfica, escolha do muro de gravidade, escolha de seis perfis, criação dos roteiros de dimensionamento no PTC Mathcad Express Prime 6.0 e por fim desenvolveu-se o aplicativo, para dispositivos móveis com sistema operacional Android, AUTOARRIMOS com a ferramenta de programação MIT App Inventor 2, pensando na agilidade e praticidade para a rotina.

O PTC Mathcad Express Prime 6.0 que é um programa com ambiente de trabalho baseado em Álgebra Computacional, resolve e documenta cálculos de modo satisfatório, Retzlaff e Contri (2011).

De acordo com Finizola et al (2014), a ferramenta MIT App Inventor 2, pode ser usado diretamente no browser e possui dois recursos principais, que são o Designer e o Blocks Editor. No Designer cria-se a interface do aplicativo, são escolhidos os componentes visuais dos aplicativos. No Blocks Editor ocorre a programação do aplicativo, por meio de blocos no estilo quebra-cabeças.

Pensando na relevância do aplicativo, observase que pode ter grande importância como ferramenta educacional. O uso de softwares, no curso de Engenharia Civil, pode tornar as aulas mais dinâmicas, criando a possibilidade de discussão sobre os dados obtidos, Martinello e Belchior (2017). Pode-se testar várias hipóteses de forma rápida e aumentando a produtividade das aulas.

Para Lobo et al (2003), o uso de taludes para manter diferenças de nível, causa grande perda de áreas, sendo o motivo de os muros de arrimo muito utilizadas nas áreas urbanas.

De acordo com o Departamento de Estradas de Rodagem do Estado de São Paulo [DER/SP] (2005), dois tipos de muros de arrimo são os mais usuais. Muros de flexão, trabalham à flexão, compostos por concreto armado ou alvenaria estrutural. Enquanto nos muros de arrimo por gravidade, o elemento estruturante é o peso próprio do muro.

Os muros de arrimo por gravidade, são utilizados para conter terraplenos de baixa altura, comparados aos muros de flexão, visto que quanto maior a solicitação maior é quantidade de material utilizado a construção, o que pode gerar uma carga muito alta e grandes gastos com material, Couto Neto (2013).
Segundo Domingues (1997), os muros de arrimo por gravidade têm a estabilidade garantida por seu peso próprio, sendo utilizados normalmente em solos com boa capacidade de suporte. Alguns dos materiais que podem constituir tais muros são concreto ciclópico, alvenaria de pedra ou solo-cimento ensacado.

O presente trabalho focou no desenvolvimento de aplicativo para o dimensionamento de muros de gravidade, constituído por concreto ciclópico.

De acordo com Vieira Júnior (2014), o termo "ciclópico" se originou na Grécia antiga, onde grandes blocos de pedra eram empilhados, sem argamassa, na construção de fortes. Ainda hoje grandes pedras são utilizadas, menores que as citadas acima, junto com argamassa, formando o concreto ciclópico, que tem como peso específico $22 \mathrm{kN} / \mathrm{m}^{3}$.

Os muros de contenção sofrem esforços provenientes do solo, tais esforços são os empuxos de terra, que podem ser divididos em ativo e passivo. $\mathrm{O}$ empuxo ativo é o esforço que o terrapleno exerce sobre o a estrutura de contenção, causando um deslocamento para longe da porção de terra a ser contida. No empuxo passivo, o terrapleno sofre compressão devido à aproximação do muro, Marangon (2018).

Para garantir a segurança da estrutura de contenção devem ser feitas verificações quanto sua estabilidade.

A verificação de segurança quanto ao tombamento do muro (Figura 1) consiste na razão de dois momentos, o momento resistente e o momento de tombamento. Tal razão resulta em um fator de segurança. O tombamento acontece considerando rotação do muro em relação ao ponto da base mais distante da porção de terra a ser contida; o momento de tombamento é causado pelo empuxo ativo. Assim o momento resistente, é relacionado à carga do muro, com rotação oposta ao tombamento, Caputo et al (2015).

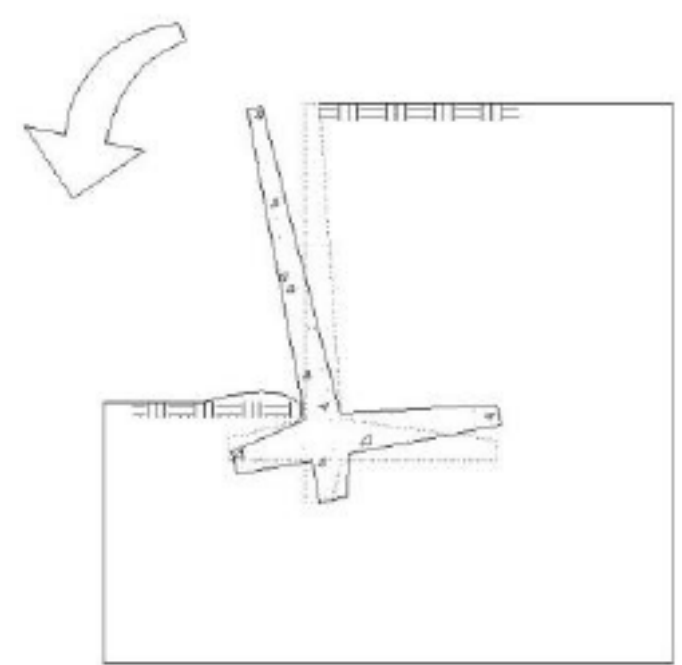

Figura 1. Representação do tombamento.

Fonte: Couto Neto, 2013. 
De acordo com Couto Neto (2013), para garantir a segurança da estrutura quanto ao tombamento o momento de resistente deve ser maior que o momento de tombamento, considerando um fator de segurança de ao menos 1,5. O que pode ser expresso por:

$$
\text { FStomb }=\frac{\text { Mres }}{\text { Mtomb }} \geq 1,5
$$

Onde:

FStomb = Fator de segurança, referente ao tombamento;

Mres $=$ Momento resistente; $\mathrm{e}$

Mtomb= Momento de tombamento.

Como é conhecido, momento pode ser obtido pelo produto de uma força por um braço de alavanca. O momento resistente (Mres), se dá pelo produto da carga proveniente do muro pela distância entre borda do elemento mais distante do terrapleno e o centro de gravidade da estrutura de contenção.

Caso o muro de arrimo possua forma geométrica que permita acúmulo de solo sobre ele, tal parcela de solo irá contribuir com o momento resistente. Os momentos podem ser calculados por partes e somados, visto que os pesos específicos de solo e muro podem ser diferentes e os carregamentos não aplicados no mesmo ponto.

O momento de tombamento é obtido pelo produto do empuxo ativo por sua altura de aplicação. Sendo tal altura equivalente a um terço da altura total de aplicação do carregamento distribuído.

A verificação de deslizamento (Figura 2) também resulta em fator de segurança, a partir de uma razão. A razão é formada basicamente pela associação de informações (empuxo passivo, peso da estrutura e ângulo de atrito do solo) com o empuxo ativo. A citada associação empuxo passivo, peso do muro e ângulo de atrito resistem à força de deslizamento causada pelo empuxo ativo, Caputo et al (2015).

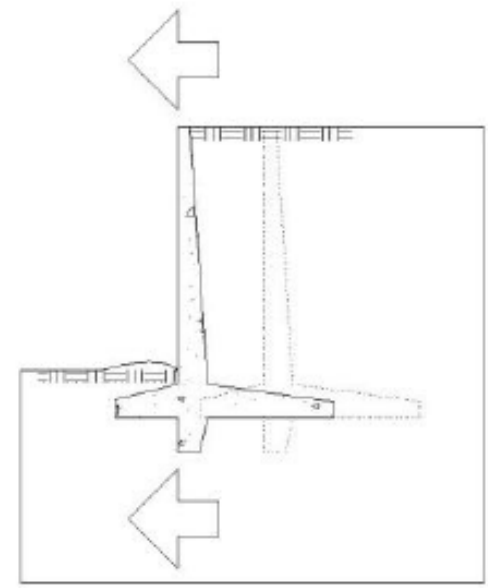

Figura 2. Representação do deslizamento.
Conforme Couto Neto (2013), adota-se o fator de segurança ao deslizamento igual a 1,5. Tal fator pode ser obtido por:

$$
\text { FSdes }=\frac{\text { Frdes }}{E a} \geq 1,5
$$

Onde:

Ep = empuxo passivo;

$\mathrm{Cm}=$ carregamento do muro; $\mathrm{e}$

$\varphi=$ ângulo interno de atrito do solo.

O carregamento do muro é equivalente a um metro linear de muro, assim pode ser obtido pelo produto da área do perfil da estrutura pelo seu peso específico.

A verificação quanto à carga do muro no solo abaixo da base é basicamente garantir se o solo possui capacidade suportar a carga gerada pelo muro. Conforme cargas são aplicadas, os solos se deformam, sendo uma verificação muito importante, Caputo et al (2015). Nesta última etapa é verificado se a carga total do muro está localizada no núcleo central da base, gerando apenas esforço de compressão e é feita comparação com a carga admissível do solo. Situação representada na figura 3.

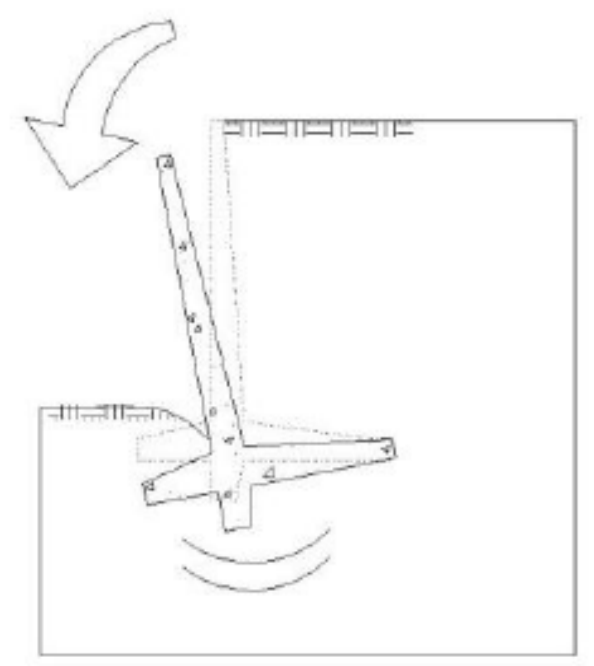

Figura 3. Representação de falha do solo devido ao carregamento do muro.

Fonte: Couto Neto, 2013.

Para que o carregamento esteja no núcleo central, provocando apenas esforços de compressão, a excentricidade, ou seja a distância entre o ponto de aplicação do carregamento e o centro da base do muro, deve ser um sexto do comprimento da base. A figura 4 ilustra a situação. 


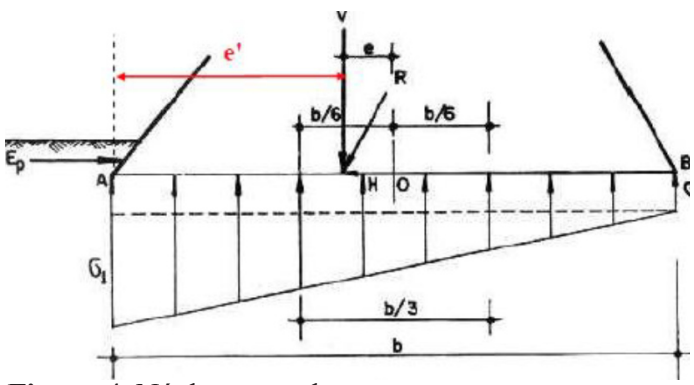

Figura 4. Núcleo central.

Fonte: Gerscovich, 2010

Para encontrar o valor da excentricidade (e), pode-se usar:

$$
e=\frac{b}{2}-e^{\prime}
$$

Onde e'representa a distância entre oa extremidade da base e ponto de aplicação do carregamento. $\mathrm{O}$ valor de e' é obtido por:

$$
e^{\prime}=\frac{\text { इmomentos }}{\mathrm{Cm}}
$$

O somatório dos momentos representa a adição entre o momento resistente e o momento de tombamento, considerando sinais diferentes devido à orientação dos momentos; enquanto que representa a carga total da estrutura de contenção.

A próxima etapa desta verificação é analisar a capacidade de suporte do solo, assim deve se fazer a comparação entre a tensão máxima provocada pelo muro e a capacidade de carga admissível do solo.

O valor de tensão máxima é obtido por:

$$
\sigma \max =\frac{\mathrm{Cm}}{\mathrm{b}} *\left(1+\frac{6 * e}{b}\right)
$$

Sendo o valor da tensão máxima, proveniente do muro, menor que o valor da capacidade de carga admissível solo, está garantida a segurança quanto ao carregamento.

Mesmo sabendo da escassez de ferramentas com o foco aqui demostrado, elas existem e cabe citar trabalhos presentes na literatura relacionados ao tema central deste artigo.

Como exemplo, o roteiro de cálculo de muro de arrimo por flexão, desenvolvido no Mathcad Prime 5.0, desenvolvido por Silva et al (2019). Tal trabalho tem resultados próximos ao aqui encontrados, como a verificação da possibilidade de se otimizar um processo de cálculo com auxílio da tecnologia.

Ou ainda, o trabalho de Couto Neto (2013), o qual apresenta um software para o dimensionamento de muros de arrimo. Software para computadores, que atendeu de modo satisfatório ao requisito de redução de tempo de projeto.

Os trabalhos citados se destacam pelo fato criarem ferramentas úteis à otimização do processo de cálculo, porém se limitam ao uso em computadores. O presente artigo apresenta um aplicativo para dispositivos móveis, que estão cada vez mais ganhando espaço nas mais diversas áreas de atuação da sociedade, facilitando $o$ acesso às informações nos mais diferentes ambientes.

\section{Metodologia}

A primeira etapa do trabalho foi a pesquisa em trabalhos acadêmicos, notas de aulas e livros; em seguida para se ter uma base de cálculo para o desenvolvimento do aplicativo para dispositivos móveis, criou-se roteiros de cálculo no software PTC Mathcad Express Prime 6.0. Para o desenvolvimento do aplicativo utilizouse a ferramenta on-line de criação MIT App Inventor 2. A Figura 5 mostra o fluxograma que representa o desenvolvimento do trabalho.

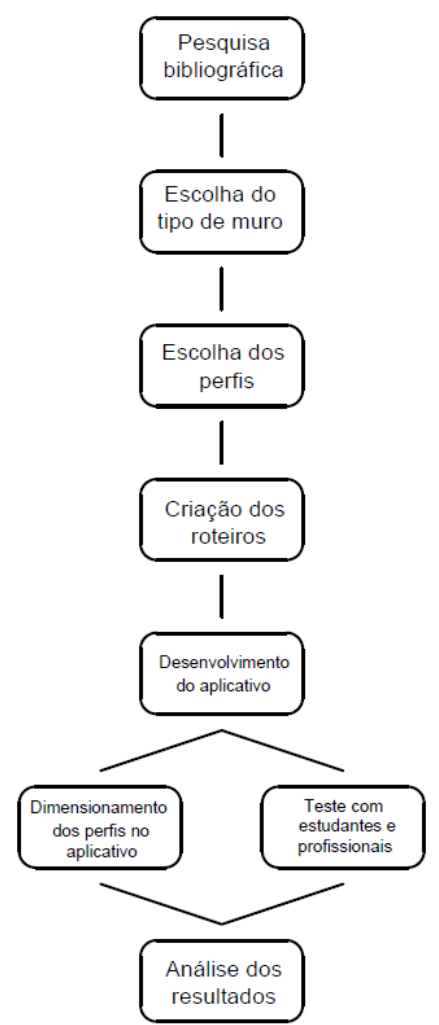

Figura 5. Fluxograma de etapas do trabalho.

Fonte: os autores, 2020.

Para o aplicativo foi considerada a hipótese de muro de gravidade, constituído de concreto ciclópico, executado em solo homogêneo. Para experimentar o funcionamento do aplicativo, foi verificada a estabilidade dos seis perfis disponibilizados, considerando uma 
mesma situação para todos, a fim de analisar o mais adequado para a situação. Além disso, profissionais e estudantes de Engenharia Civil testaram o aplicativo.

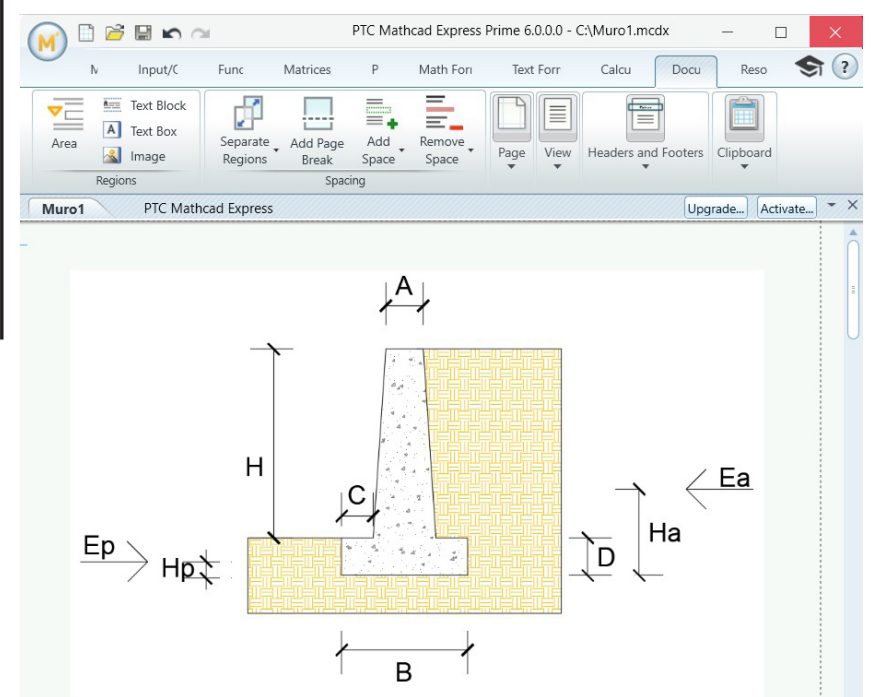

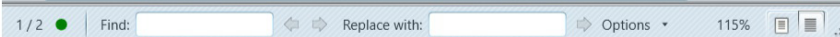

Figura 6. Imagem do perfil 1 no roteiro de cálculo no PTC Mathcad Express Prime 6.0; A, B, C, D e H são dimensões do perfil, Ep representa empuxo passivo, Ea representa empuxo ativo, Hp e Há são as alturas onde incidem os valores de empuxo.

Fonte: os autores, 2020.

O roteiro de cálculo desenvolvido no software PTC Mathcad Express Prime 6.0 apresenta logo no seu início ilustração do perfil, onde são indicadas suas dimensões e os dados referentes ao empuxo, como mostra a Figura 6. Em seguida o roteiro sugere a inserção de dados necessários ao cálculo, como as dimensões desejadas, as informações de empuxo e as informações sobre o solo, ilustrado na Figura 7.

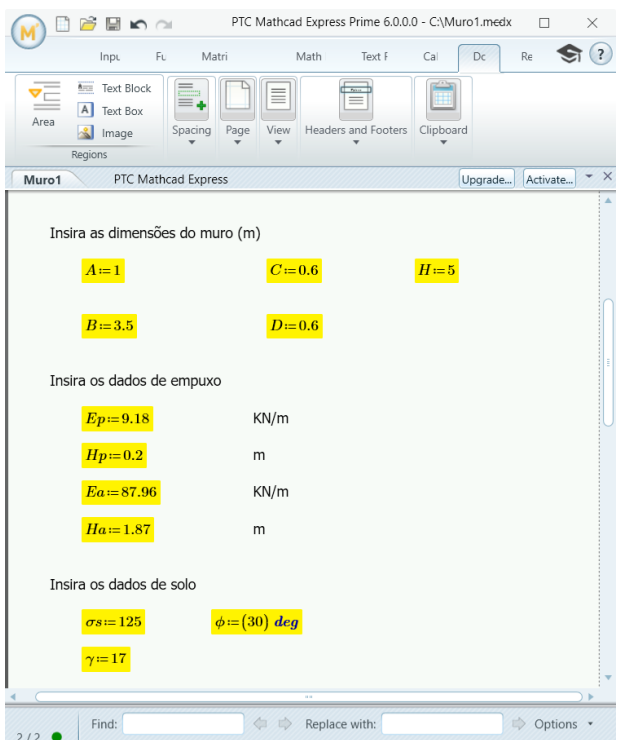

Figura 7. Inserção de dados no roteiro de cálculo. Os dados de solo são carga admissível, peso específico e ângulo de atrito.

Fonte: os autores, 2020.
Após a inserção de dados, tendo o roteiro de cálculo completamente montado, são efetuados automaticamente os cálculos das verificações de segurança, de acordo com as dimensões do muro e seu peso, considerando a constituição de concreto ciclópico e as características do solo a ser contido, que além disso suporta a carga do muro.

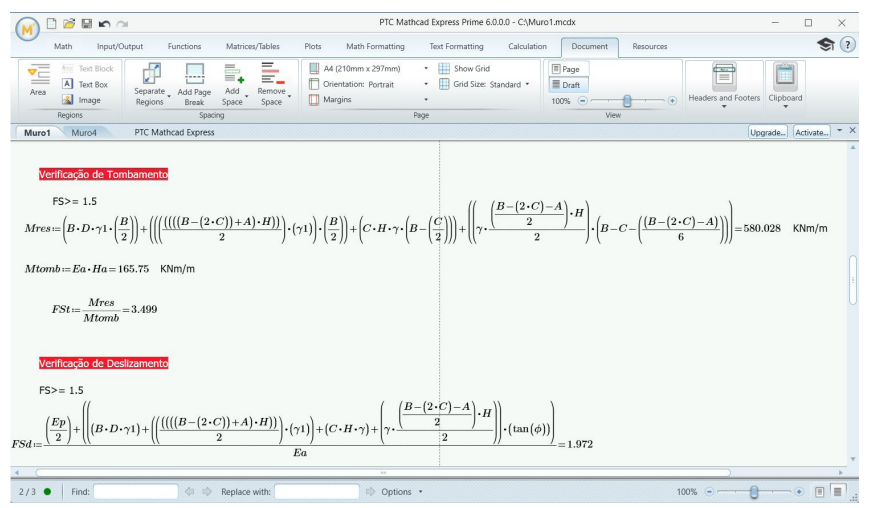

Figura 8. Verificações de tombamento e deslizamento, do perfil 1, no roteiro de cálculo.

Fonte: os autores, 2020.

Foram realizadas as verificações de segurança com relação ao tombamento e ao deslizamento (Figura 8) do muro e uma terceira verificação relativa à carga do muro aplicada no solo (Figura 9).

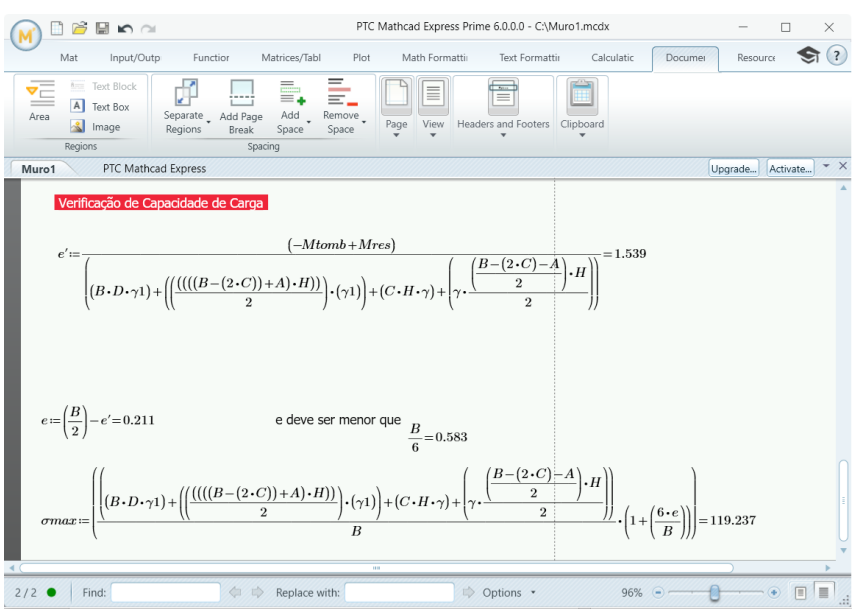

Figura 9. Verificações quanto à carga, do perfil 1, no roteiro de cálculo.

Fonte: os autores, 2020.

Após a escolha dos seis perfis que seriam disponibilizados e a montagem dos respectivos roteiros de cálculo, deu-se se início à criação do aplicativo. Para o desenvolvimento do aplicativo, que foi nomeado de AUTOARRIMOS, fez-se uso da ferramenta MIT App Inventor 2.

A ferramenta MIT App Inventor 2 possui uma interface simples e amigável, que permite a criação de aplicativos sem que haja a necessidade de fazer 
a digitação de linhas de código de programação. Toda a lógica de programação está disponível em blocos, desde a criação da interface das páginas até as configurações das funções.

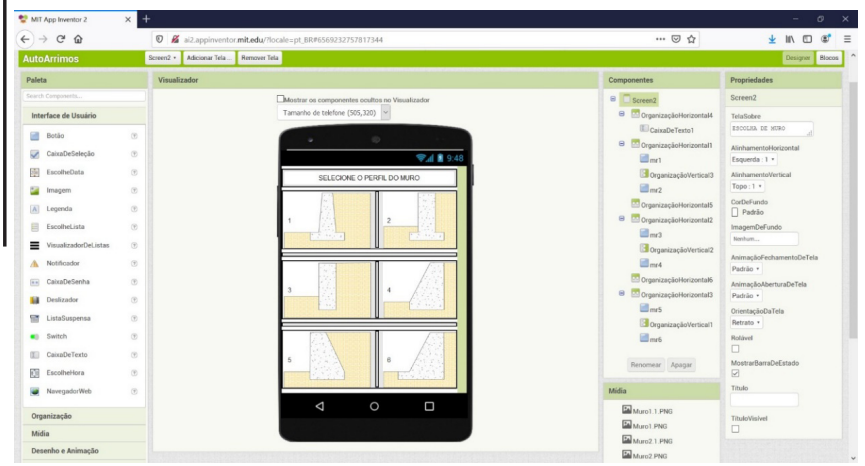

Figura 10. Interface da página de seleção dos perfis, ainda na ferramenta MIT App Inventor 2.

Fonte: os autores, 2020.

Para a criação das interfaces das páginas basta arrastar os objetos desejados (imagens, caixas de texto, botões, etc) e configurar o que deseja visualmente, como formato, tamanho, cor e texto. A Figura 10 mostra a interface da página de seleção dos perfis, ainda na ferramenta MIT App Inventor 2.

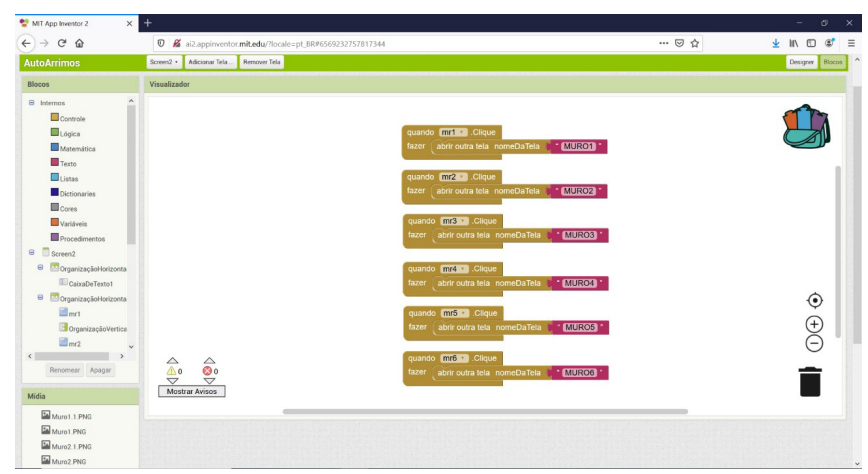

Figura 11. Configuração das funções da página de seleção dos perfis, na ferramenta MIT App Inventor 2.

Fonte: os autores, 2020.

Após a criação da parte visual das páginas, iniciou-se a configuração das funções, como mostra a Figura 11, que ilustra a configuração da página citada anteriormente. Nesta etapa basta, assim como na criação da interface, arrastar as funções e posicioná-las como peças de quebracabeças, organizando de acordo com a lógica de programação. A ferramenta MIT App Inventor 2 possui a possibilidade de criação de variáveis e de aplicação de cálculos matemáticos, o que possibilitou a aplicação do roteiro desenvolvido em fase anterior.

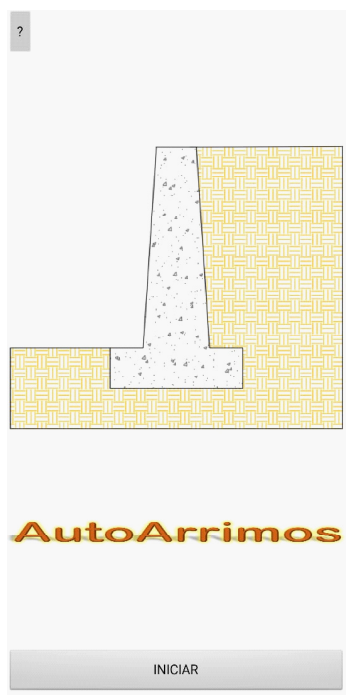

Figura 12. Página inicial do aplicativo.

Fonte: os autores, 2020.

O aplicativo completo apresenta uma interface bem simples, facilitando a utilização, foi estruturado da seguinte forma: página inicial (Figura 12), que possui botões para a página de informações (Figura 13) e para página de seleção de perfis (Figura 14). Por fim é aberta a página de verificação de estabilidade de cada perfil, de acordo com a escolha na tela anterior.
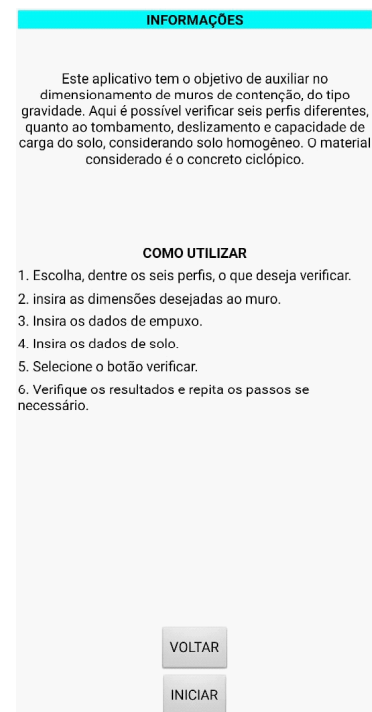

Figura 13. Página de informações.

Fonte: os autores, 2020

A página de informações traz um breve texto que situa o usuário quanto à funcionalidade do aplicativo. O texto expressa ao usuário o objetivo do aplicativo, que é auxiliar no dimensionamento 
de muros de arrimo por gravidade, efetuando as verificações quanto ao tombamento, deslizamento e carregamento.

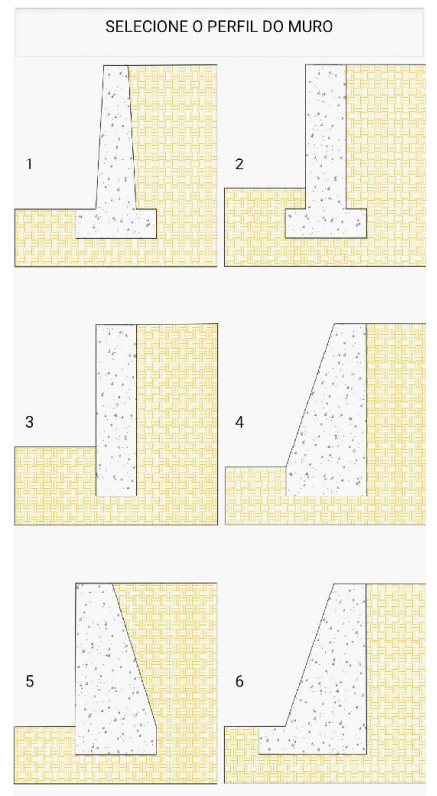

Figura 14. PPágina de seleção de perfis

Fonte: os autores, 2020.

A página também faz uma descrição dos passos a serem seguidos para a utilização do aplicativo, a fim de esclarecer possíveis dúvidas dos usuários.

Ao escolher o perfil que se deseja verificar, na página de seleção, abre-se a tela relacionada. Cada página de perfil exibe imagem do perfil, recomendações de dimensões, solicita a inserção de dados (as mesmas do roteiro); abaixo possui o botão verificar, quando clicado é exibido os resultados de verificação no fim da página. Abaixo dos resultados existem dois botões, o primeiro retorna à página de seleção de perfis e o último finaliza o aplicativo. A figura 15 mostra a página do perfil 1 , antes da inserção de dados e realização das verificações.

Após o término da produção do aplicativo, foi o momento de testar e obter respostas sobre o funcionamento do aplicativo na prática. Dois foram os modos escolhidos para o teste.

O primeiro teste executado foi a realização da verificação de estabilidade dos seis perfis disponíveis no aplicativo, considerando as mesmas condições do solo para todos eles. O teste foi realizado com o objetivo buscar possíveis falhas de funcionamento do aplicativo e ainda com os resultados obtidos foi possível compará-los.

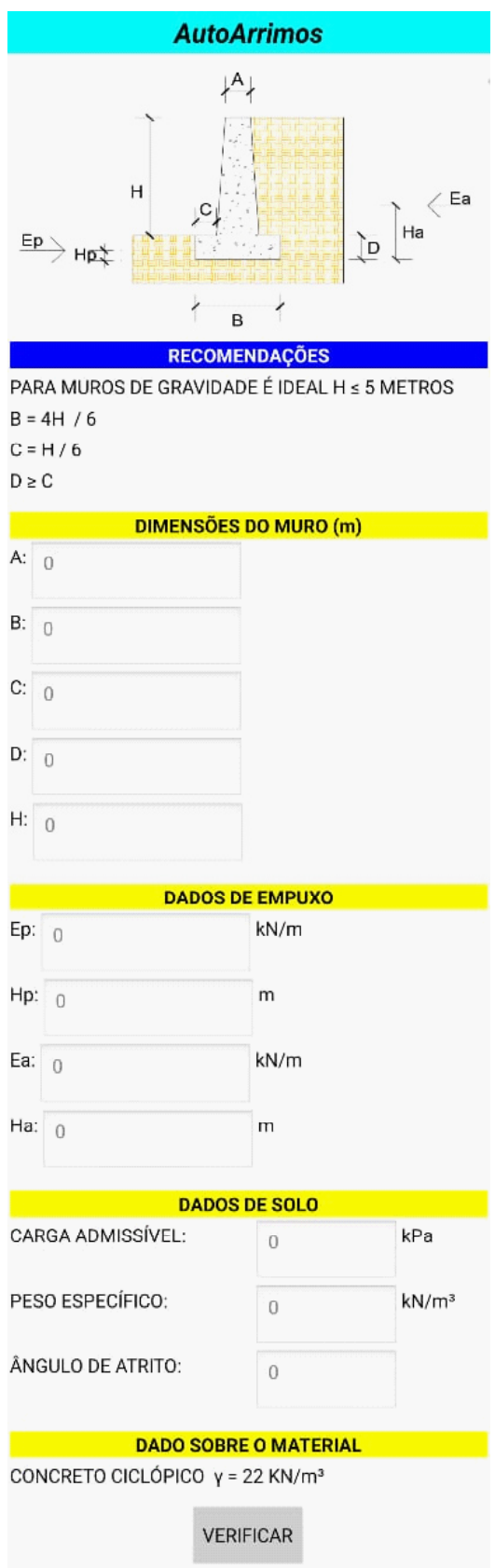

Figura 15. Página do perfil 1 antes dos cálculos.

Fonte: os autores, 2020.

Foi considerado solo arenoso, sem coesão, com peso específico de $17 \mathrm{kN} / \mathrm{m}^{3}$, ângulo de atrito de $30^{\circ}$, carga admissível igual a $125 \mathrm{kPa}$ e terrapleno a ser contido com altura de 5 metros. Além disso, adotou-se hipótese de solo homogêneo e sem a presença de lençol freático.

Foram considerados todos os muros com altura igual a 5,6 metros, considerando 5 metros do terrapleno e 60 centímetros abaixo do nível do solo. Tendo em mãos os dados fundamentais, fezse o cálculo dos empuxos, bem como das alturas de aplicação. 
Vale ressaltar que o aplicativo deve ser utilizado por usuários com conhecimentos sobre a área, visto que o cálculo do empuxo bem como sua altura de aplicação deve ser calculado pelo usuário e inserido para as verificações de estabilidade.

A seguir, os cálculos de empuxo:

$$
\begin{gathered}
K a=\operatorname{tg}^{2}\left(45-\frac{30}{2}\right)=0,333 \\
E a=\frac{1}{2} * 17 * 5,6^{2} * 0,333=87,964 k N \\
h a=5,6 * \frac{1}{3}=1,866 \mathrm{~m} \\
K p=\operatorname{tg}{ }^{2}\left(45+\frac{30}{2}\right)=3 \\
E p=\frac{1}{2} * 17 * 0,6^{2} * 3=9,18 \mathrm{kN} \\
h p=0,6 * \frac{1}{3}=0,2 \mathrm{~m}
\end{gathered}
$$

O quadro 1 resume os valores obtidos, que foram dados de entrada para a verificação de estabilidade dos seis perfis de muro, considerando a mesma situação hipotética.

Quadro1. Informações de empuxo.

\begin{tabular}{|c|c|c|}
\hline Informações & Estado ativo & Estado passivo \\
\hline Coeficiente & 0,33 & 3 \\
\hline Empuxo & $87,96 \mathrm{kN}$ & $9,18 \mathrm{kN}$ \\
\hline Altura de aplicação & $1,87 \mathrm{~m}$ & $0,2 \mathrm{~m}$ \\
\hline \multicolumn{3}{|c|}{ Fonte: os autores, 2020.}
\end{tabular}

O segundo teste consistiu na disponibilização do aplicativo para profissionais e estudantes de Engenharia Civil. Após a utilização, responderam um questionário para registrar suas impressões. Além de responder as perguntas, os usuários que realizaram o teste deixaram sugestões e opiniões.

O questionário foi elaborado e disponibilizado por meio de formulário on-line, onde cada usuário pôde fazer sua avaliação no momento que considerasse mais adequado. O aplicativo foi testado por 8 usuários, mesmo não sendo um número tão alto, serviu como meio de verificar uma percepção diferente dos autores, onde poderiam surgir pontos não pensados na elaboração do aplicativo.

\section{Resultados e discussão}

$\mathrm{O}$ teste feito com usuários, profissionais e estudantes de Engenharia Civil, trouxe bons resultados e ainda sugestões. O questionário foi composto por 5 questões e um espaço para sugestões.

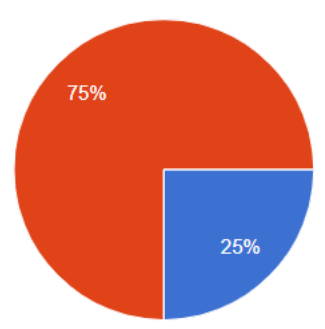

Engenheiro(a) Civil. Estudante de Engenharia.

Figura 16. Perfil dos usuários no teste.

Fonte: os autores, 2020.

A primeira questão traz o perfil dos usuários, como mostrado na Figura 16; o segundo ponto levantado no questionário foi a percepção do usuário com relação ao layout do aplicativo, Figura 17; o terceiro tópico foi a consulta sobre a satisfação quanto à página de informações, ilustrado na Figura 18; o quarto ponto questionou quanto aos resultados obtidos no aplicativo (Figura 19) e o último aspecto se refere ao tempo de verificação, Figura 20.

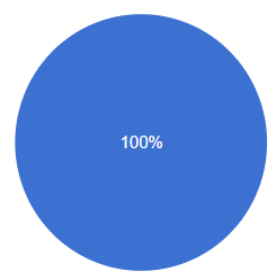

Intuitivo e de fácil usabilidade. Complicado, dificultando o uso Não permite compreender como usar o aplicativo

Figura 17. Respostas sobre o layout.

Fonte: os autores, 2020.

O aplicativo foi testado por usuários com conhecimentos como Mecânica dos Solos e das Rochas, a fim de compreender os conceitos dos cálculos e dos dados iniciais, tais como empuxo ativo e passivo. Os softwares devem servir como ferramenta, facilitando e

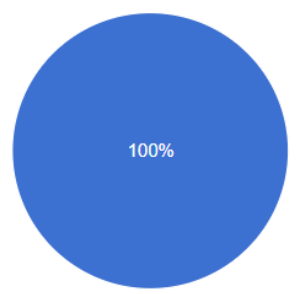

Possui todas as informações necesárias.

Informações não estão claras - Faltam informações

Figura 18. Respostas sobre a página de informações.

Fonte: os autores, 2020 
agilizando os processos, porém a responsabilidade dos dados é de quem opera a ferramenta.

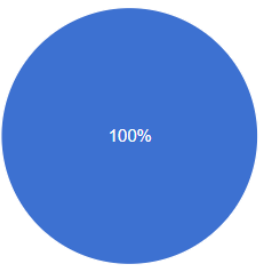

Resultados satisfatórios.

Resultados parcialmente satisfatórios Resultados insatisfatórios

Figura 19. Respostas sobre os resultados das verificações.

Fonte: os autores, 2020.

De acordo com as respostas dos usuários, o aplicativo atingiu resultados positivos e foram deixadas sugestões. A primeira sugestão foi referente às limitações da versão atual do aplicativo, foi sugerido a inclusão da consideração de solos heterogêneos e presença de nível freático. Foi sugerido ainda a criação de versão do aplicativo AUTOARRIMOS para o sistema operacional IOS.
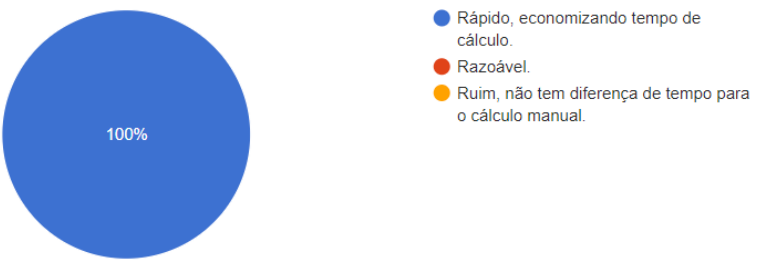

cálculo manual

Figura 20. Respostas sobre o tempo de realização das verificações.

Fonte: os autores, 2020.

Considerando a situação hipotética apresentada anteriormente, foi realizado o outro teste, que consistiu na verificação dos seis perfis disponíveis no aplicativo. As Figuras, de 21 a 26, a seguir mostram as páginas de cada perfil, no aplicativo, após o cálculo.

As figuras, de 27 a 32, a seguir apresentam os perfis com as indicações de dimensões e ponto de aplicação de empuxo, os valores das dimensões estão representados no Quadro 2. No mesmo quadro estão representados os resultados das verificações de estabilidade, são eles os valores de fator de segurança com relação ao tombamento, fator de segurança com relação deslizamento, o valor da tensão máxima do muro e ainda se a carga está aplicada no núcleo central da base do muro.

Fazendo uma primeira análise do teste, percebeuse que o aplicativo efetuou a verificação de estabilidade dos seis perfis sem apresentar erros ou problemas.

Quanto aos fatores de segurança, apenas os perfis 4 e 6 não atingiram valor satisfatório, para o fator de segurança em relação ao deslizamento.

Com relação à tensão máxima, os perfis 1,2 e 3 apresentaram valores menores que a tensão admissível do solo, enquanto que os demais perfis ultrapassaram o valor.

Os perfis 4, 5 e 6 não apresentaram carregamento concentrado no núcleo central da base.

Completando a análise, considerando que todos os perfis foram verificados sob as mesmas condições, pôde-se definir que de acordo com a comparação que o perfil 3 apresentou melhores resultados, visto que atendeu todos os critérios e ainda apresentou menor tensão máxima.

A tensão máxima do perfil 3 , sendo a menor, o torna o mais viável, sendo que o gasto com material é menor se comparado aos demais e provoca menos esforços ao solo.

Cabe ressaltar que a mudança de dimensões pode acarretar variações dos resultados, assim os valores obtidos não são absolutos e sim relativos aos valores inseridos. Cabe ao usuário fazer a análise dos dados e refinar os resultados de acordo com que se obtém e o que se deseja.

Assim, por exemplo, perfis que apresentam altos valores de fator de segurança e tensão máxima podem ter dimensões reduzidas, causando redução dos valores resultantes, buscando fatores de segurança ainda satisfatórios, porém tensões mais adequadas à realidade do solo.

\section{Considerações Finais}

Pôde-se concluir com os resultados observados que a utilização do roteiro de cálculo desenvolvida no PTC Mathcad Express Prime 6.0 e a criação de aplicativo na ferramenta MIT App Inventor 2 foi uma boa combinação. Observou-se ainda que o aplicativo se mostrou viável, possui simples utilização, chegou a resultados de modo significativamente mais rápido que o modo manual, atendendo ao objetivo do trabalho.

O aplicativo, além de ser uma ferramenta para cálculo, pode ter uma função educacional; sendo utilizado, em sala de aula, no curso de Engenharia Civil. O AUTOARRIMOS pode ser útil na resolução de exercícios, servindo como um verificador do cálculo manual, visto a facilidade de manuseio e o fato de ser desenvolvido para dispositivos móveis. Pode facilmente ser utilizado em sala de aula, devido ao fato de necessitar apenas de um smartphone ou tablet.

De acordo com as respostas dos usuários, que testaram o aplicativo, notou-se que o aplicativo atendeu às expectativas de uso, cumprindo o que se propôs.

Porém cabe melhoria, criando objetivos para realizações futuras de aperfeiçoamento do aplicativo. Devido ao fato de estar disponível apenas para o sistema operacional Android, o desenvolvimento de uma versão 


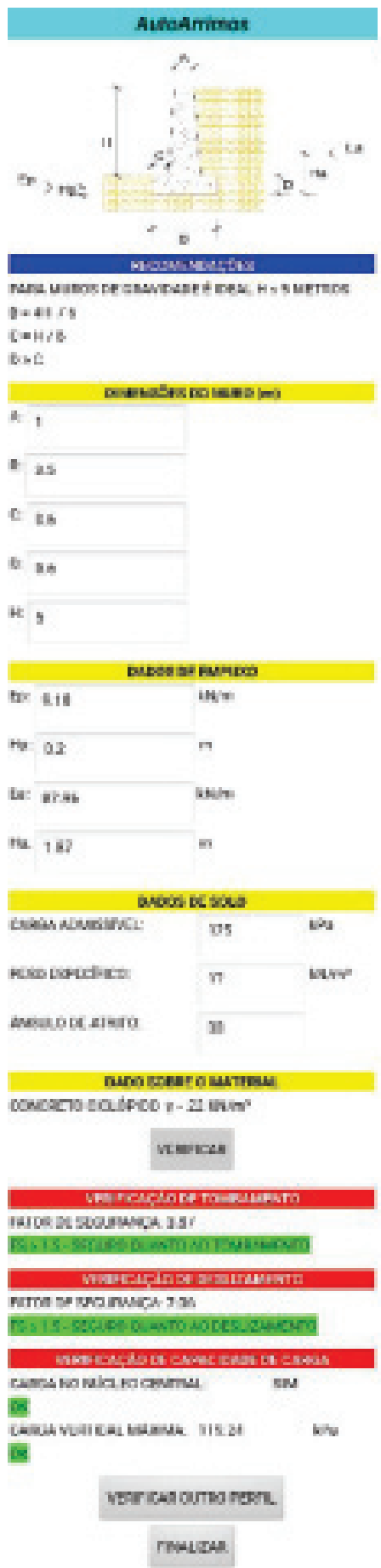

Figura 21. Verificação do perfil 1. Fonte: os autores, 2020

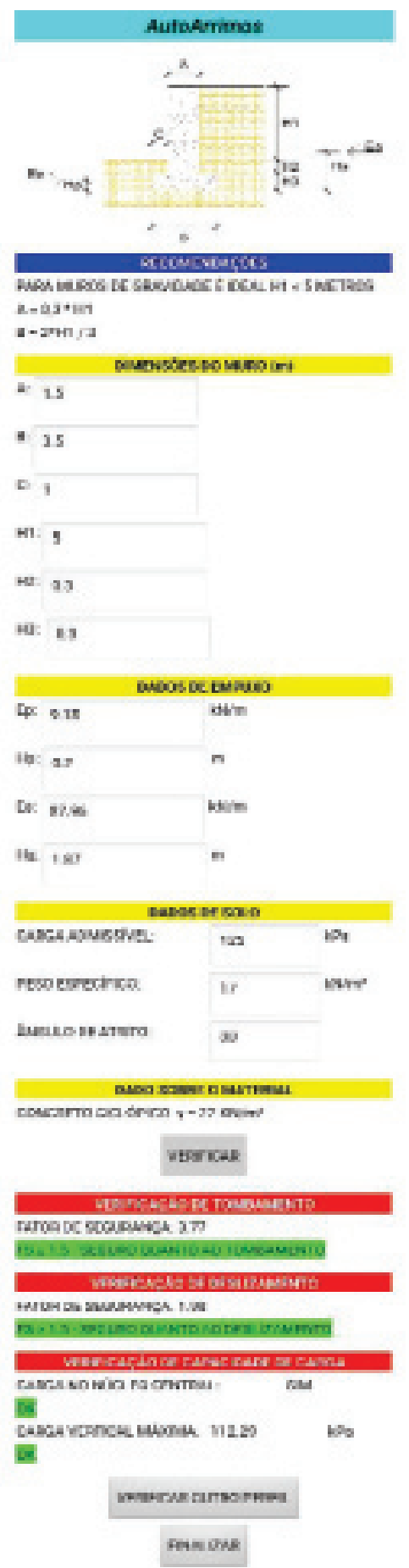

Figura 22. Verificação do perfil 2. Fonte: os autores, 2020.

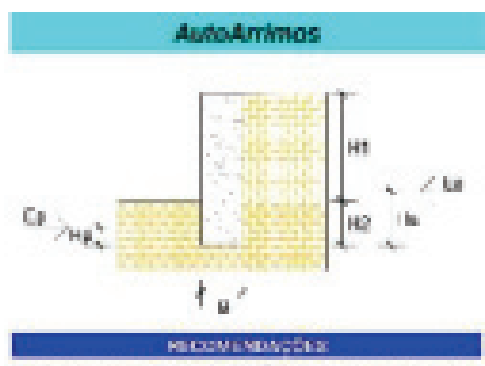

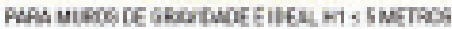
$0 * 0 z+i$ in

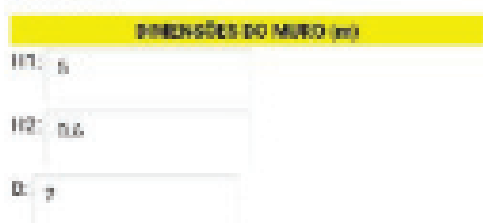

\begin{tabular}{|c|c|c|}
\hline & & DEOSG DCRECUES \\
\hline 45. & 918 & ave \\
\hline $\mathrm{Ha}$ & e2 & m \\
\hline Ea: & was & bhe \\
\hline $\mathrm{Ha}$ & $1 \times 0$ & m \\
\hline
\end{tabular}

\begin{tabular}{|c|c|c|}
\hline \multicolumn{3}{|c|}{ choos uc soco } \\
\hline CIDC, Noverinn: & 125 & n \\
\hline rexn nopriws: & 17 & mere \\
\hline incue dar aram: & $\boldsymbol{n}$ & \\
\hline
\end{tabular}

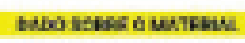

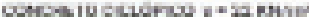

$$
\text { MERTM }
$$

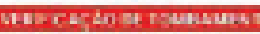

rasouse socunawsa 1 so

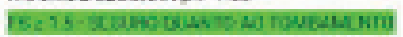

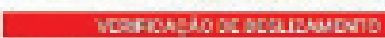

Foroa oc socisowich 1.87

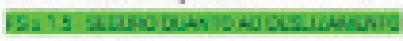

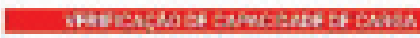

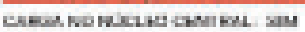

Es

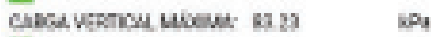

es

Wearcek buino pust
rmuleer

Figura 23. Verificação do perfil 3. Fonte: os autores, 2020 


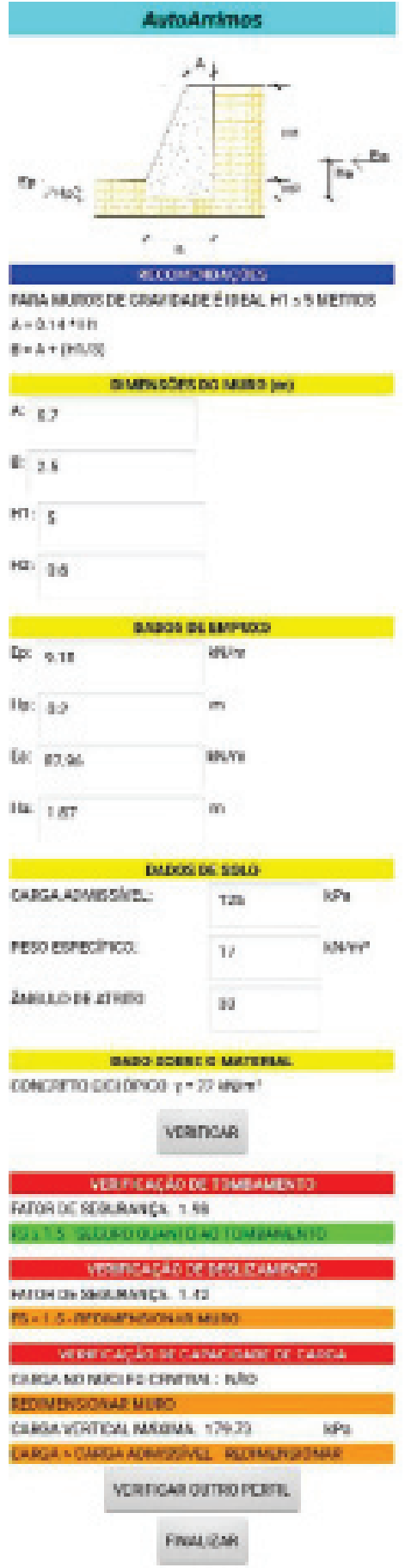

Figura 24. Verificação do perfil 4.

Fonte: os autores, 2020.

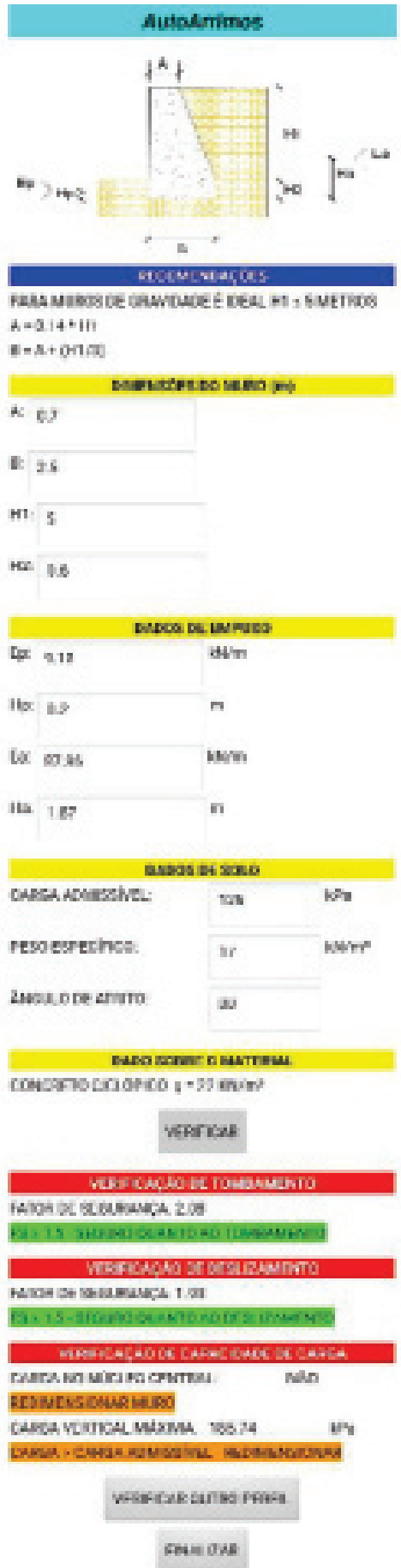

Figura 25. Verificação do perfil 5.

Fonte: os autores, 2020.

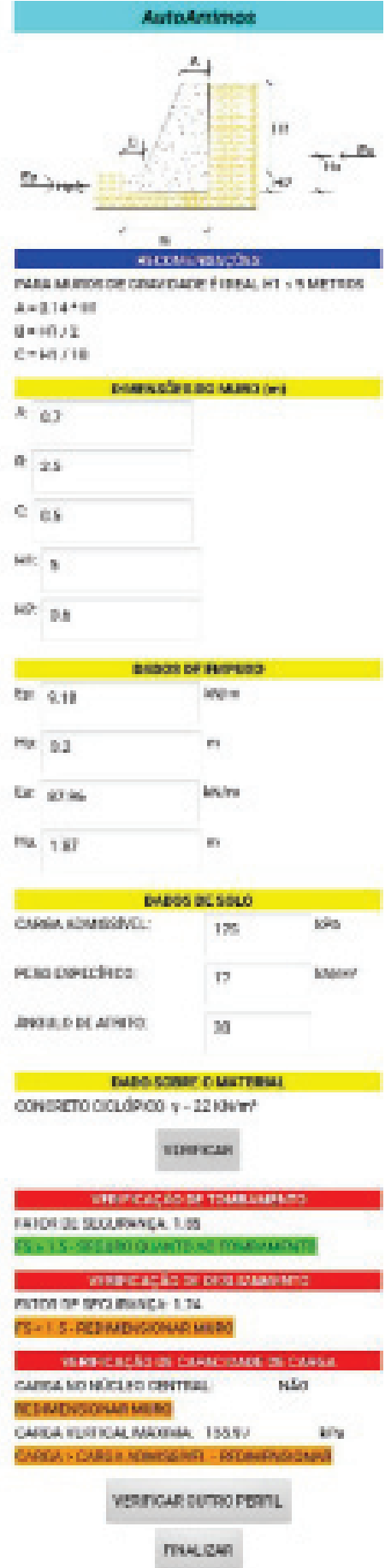

Figura 26. Verificação do perfil 6.

Fonte: os autores, 2020. 


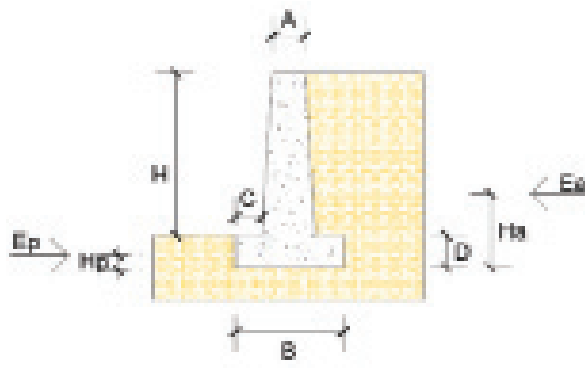

Figura 27. Perfil 1 com indicação de dimensões.

Fonte: os autores, 2020.

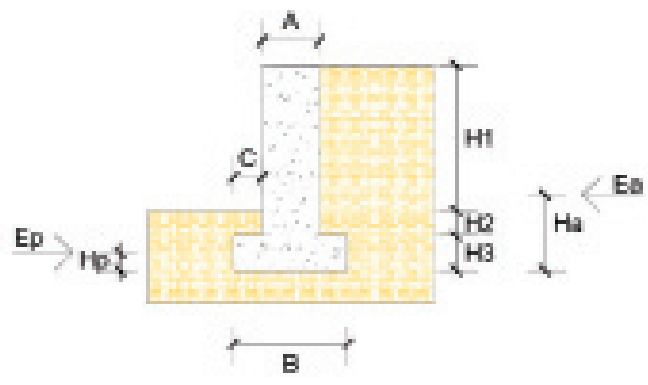

Figura 28. Perfil 2 com indicação de dimensões.

Fonte: os autores, 2020

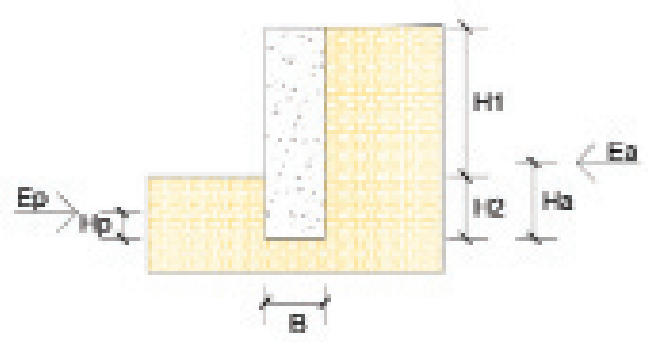

Figura 29. Perfil 3 com indicação de dimensões.

Fonte: os autores, 2020.

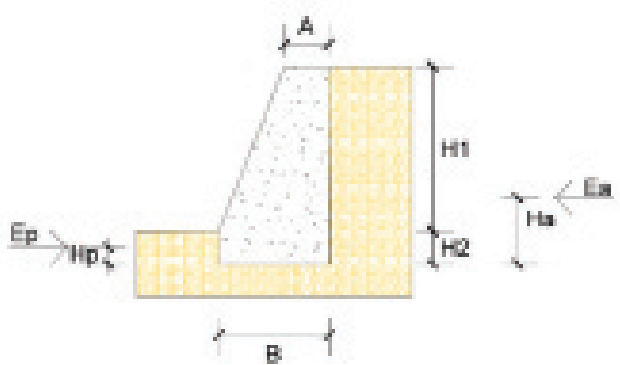

Figura 30. Perfil 4 com indicação de dimensões.

Fonte: os autores, 2020

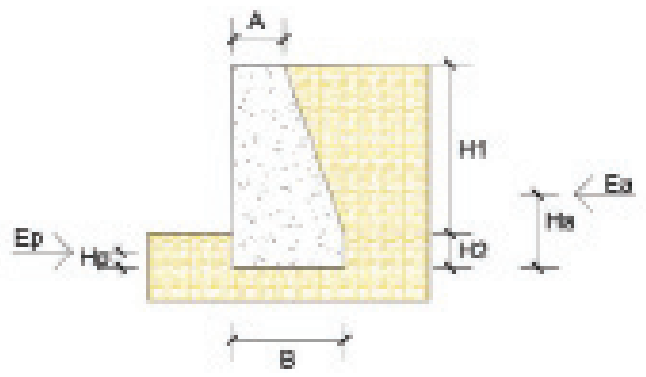

Figura 31. Perfil 5 com indicação de dimensões.

Fonte: os autores, 2020

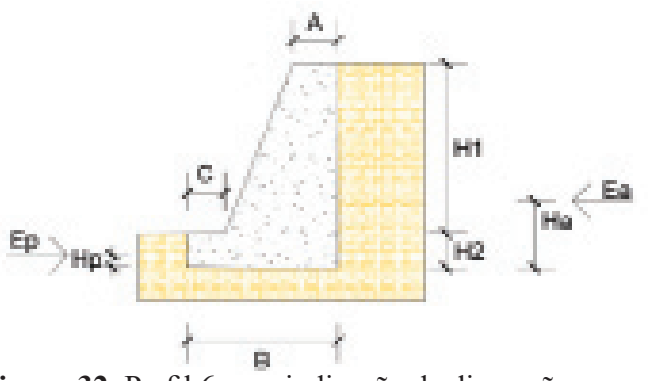

Figura 32. Perfil 6 com indicação de dimensões.

Fonte: os autores, 2020 
Quadro 2. Resultados das verificações.

\begin{tabular}{|c|c|c|c|c|c|c|}
\hline \multirow[b]{2}{*}{ Perfil } & \multirow{2}{*}{\multicolumn{2}{|c|}{$\begin{array}{l}\text { Dimensões } \\
\text { (m) }\end{array}$}} & \multicolumn{4}{|c|}{ Resultados } \\
\hline & & & FStomb & FSdes & Carga no núcleo & $\begin{array}{l}\text { Tensào } \\
\text { máxima }\end{array}$ \\
\hline \multirow{5}{*}{1} & A & 1 & \multirow{5}{*}{3,87} & \multirow{5}{*}{2,06} & \multirow{5}{*}{$\operatorname{sim}$} & \multirow{5}{*}{119,24} \\
\hline & B & 3,5 & & & & \\
\hline & $\mathrm{C}$ & 0,6 & & & & \\
\hline & $\mathrm{D}$ & 0,6 & & & & \\
\hline & $\mathrm{H}$ & 5 & & & & \\
\hline & & & & & & \\
\hline \multirow{6}{*}{2} & A & 1,5 & \multirow{6}{*}{3,77} & \multirow{6}{*}{1,98} & \multirow{6}{*}{$\operatorname{sim}$} & \multirow{6}{*}{112,29} \\
\hline & B & 3,5 & & & & \\
\hline & $\mathrm{C}$ & 1 & & & & \\
\hline & $\mathrm{H} 1$ & 5 & & & & \\
\hline & $\mathrm{H} 2$ & 0,3 & & & & \\
\hline & $\mathrm{H} 3$ & 0,3 & & & & \\
\hline & & & & & & \\
\hline \multirow{3}{*}{3} & $\mathrm{HI}$ & 5 & \multirow{3}{*}{1,5} & \multirow{3}{*}{1,67} & \multirow{3}{*}{$\operatorname{sim}$} & \multirow{3}{*}{83,23} \\
\hline & $\mathrm{H} 2$ & 0,6 & & & & \\
\hline & B & 2 & & & & \\
\hline & & & & & & \\
\hline \multirow{4}{*}{4} & $\mathrm{~A}$ & 0,7 & \multirow{4}{*}{1,98} & \multirow{4}{*}{1,42} & \multirow{4}{*}{ não } & \multirow{4}{*}{179,73} \\
\hline & $\mathrm{B}$ & 2,5 & & & & \\
\hline & $\mathrm{H} 1$ & 5 & & & & \\
\hline & $\mathrm{H} 2$ & 0,6 & & & & \\
\hline & & & & & & \\
\hline \multirow{4}{*}{5} & $\mathrm{~A}$ & 0,7 & \multirow{4}{*}{2,08} & \multirow{4}{*}{1,93} & \multirow{4}{*}{ não } & \multirow{4}{*}{185,74} \\
\hline & $\mathrm{B}$ & 2,5 & & & & \\
\hline & $\mathrm{H} 1$ & 5 & & & & \\
\hline & $\mathrm{H} 2$ & 0,6 & & & & \\
\hline & & & & & & \\
\hline \multirow{5}{*}{6} & A & 0,7 & & & & \\
\hline & B & 2,5 & & & & \\
\hline & $\mathrm{C}$ & 0,5 & 1,85 & 1,24 & não & 155,97 \\
\hline & $\mathrm{H} 1$ & 5 & & & & \\
\hline & $\mathrm{H} 2$ & 0,6 & & & & \\
\hline
\end{tabular}

Fonte: os autores, 2020.

para o sistema operacional IOS tornou-se uma meta.

Outro objetivo para aperfeiçoamento do aplicativo é considerar solos heterogêneos e com a presença de nível freático.

Ainda têm-se o intuito de contemplar maior parte do processo de dimensionamento de muros de arrimo, incluído o cálculo dos valores de empuxo; além de disponibilizar a verificação dos muros de gabião.

O último objetivo para melhoria do aplicativo é a inclusão do dimensionamento de muros de arrimo, por flexão.

\section{Referências}

Caputo, H. P.; Caputo, A. N.; Rodrigues, J. M. A. (2015). Mecânica dos solos e suas aplicações: mecânica das rochas, fundações e obras de terra: volume 2 - 7. ed. - Rio de Janeiro: LTC.

Couto Neto, P. C. D. (2013). Desenvolvimento de software para cálculo de muros de arrimo. Centro Universitário de Brasília. TCC. Brasília, DF, Brasil.

da Silva, J. T.; Monteiro, A.S.; Gomes, G. J. C.; de Carvalho, M. B. (2019) Dimensionamento de muros de arrimo submetidos à flexão com a utilização do software Mathcad 5.0: Roteiro automatizado didático. Revista Teccen, 12 (2): 11-22.

Departamento de Estradas de Rodagem do Estado de São Paulo. (2005). Projeto de Muro de Arrimo. São Paulo. Disponível em: ftp://ftp.sp.gov.br/ ftpder/normas/IP-DE-C00-005 A.pdf 
Domingues, P. C. (1997). Indicações para projeto de muros de arrimo em concreto armado. Universidade de São Paulo. Dissertação. São Carlos, SP, Brasil.

Finizola, A. B., Raposo, E. H. S., Pereira, M. B. P. N., Gomes, W. S., de Araújo, A. L. S. O., \& Souza, F. V. C. (2014). O ensino de programação para dispositivos móveis utilizando o MIT-App Inventor com alunos do ensino médio. In Anais do Workshop de Informática na Escola (Vol. 20, No. 1, p. 337).

Gerscovich, D. (2010). Apostila estruturas de contenção muros de arrimo. Rio de Janeiro. Recuperado em 11 setembro, 2019, de: http://www.eng.uerj. br/ denise/pdf/muros.pdf

Inc., P. (2019). Matchcad Express Prime (Versão 6.0.) [Software]. Boston, Massachusetts, EUA: PTC.

Lobo, A. S.; Ferreira, C. V., \& Renofio, A. (2003). Muros de arrimo em solos colapsíveis provenientes do arenito Bauru: problemas executivos e influência em edificações vizinhas em áreas urbanas. Acta Scientiarum. Technology, 25(2), 169-177

Marangon, M. (2018). Notas de Aulas: Mecânica dos Solos II. Universidade Federal de Juiz de Fora (UFJF): Faculdade de Engenharia-NuGeo/Núcleo de Geotecnia. Disponível em: http://www.ufjf.br/nugeo/files/2013/06/ MARANGON-2018-Cap\%C3\%ADtulo-06-Empuxos-de-Terra-20181.pdf. Acesso em, 22/03/2020.

Martinello, L. H. Z.; \& Belchior, I.M.R.M. (2017). Desenvolvimento de software educacional para determinação de empuxos de terra e estabilidade de muros de gravidade. Artigo submetido à Universidade do Extremo Sul Catarinense para obtenção de bacharelado em Engenharia Civil. Criciúma, $\mathrm{SC}$, Brasil.

Massachusetts Institute of Technology (2013). App Inventor (Versão 2) [Ferramenta on-line]. Cambridge, MA, EUA: MIT. Disponível em: http:// appinventor.mit.edu/.

Retzlaff, E., \& Contri, R. D. F. F. (2011). Produção de vídeoaulas com Camtasia Studio e software Mathcad - Recursos para o ensino/aprendizagem da matemática. Revista ENCITEC, 1(1), 128-137.

Vieira Júnior, G. T. (2014). Estudo de comparativo de viabilidade econômica entre muros de arrimo de gravidade, utilizando o gabião comparando com concreto ciclópico. TCC. Centro Universitário do Sul de Minas. Varginha, MG, Brasil. 